\title{
Geometric Mean Algorithms Based on Harmonic and Arithmetic Iterations
}

\author{
Ben Jeuris and Raf Vandebril
}

KU Leuven, Dept. of Computer Science, 3001 Leuven(Heverlee), Belgium

\{ben.jeuris, raf.vandebril\}@cs.kuleuven. be

\begin{abstract}
The geometric mean of a series of positive numbers $a_{1}, \ldots, a_{n}$ is defined as the $n$th root of its product: $\sqrt[n]{a_{1} \cdots a_{n}}$. Generalizing this concept to positive definite matrices is not straightforward due to the noncommutativity. Based on a list of desired properties -the ALM-listinitially some recursive algorithms were proposed. Unfortunately, these algorithms were typically quite time consuming. A novel optimization based approach leads to the concept of the Karcher mean and is nowadays favored as the generalization towards matrices of the geometric mean.

It is not so well-known that one can design for two scalars an algorithm quadratically convergent to the geometric mean, solely relying on arithmetic and harmonic means. The procedure is straightforward, compute the arithmetic and harmonic mean, and iterate this procedure with these newly computed means.

Apparently, this procedure also works for two matrices. In these notes, we will propose a whole series of fast possible generalizations, and conduct numerical experiments with these algorithms. Unfortunately most of them do not satisfy all imposed ALM-constraints, but on the other hand, they converge rapidly and approximate the Karcher mean. As such they can be used to initiate manifold optimization procedures for computing the Karcher mean.
\end{abstract}

\section{Introduction}

The idea of the algorithms proposed in this article is not to satisfy all ALMproperties [1 in a full theoretical sense. We do, however, aim at providing numerical means, up to a certain accuracy. More precisely, suppose that for a practical application only 4 accurate digits are required, and one of the algorithms proposed in this article is capable of computing a matrix, equal to the actual Karcher mean up to four accurate digits. Then this mean can replace the Karcher mean in the application, increasing the computational efficiency.

\section{Arithmetic-Harmonic Mean for Two Elements}

\subsection{The Scalar Case}

Let $a_{1}, a_{2}$ be positive numbers and let us define the harmonic, arithmetic, and geometric means as follows:

F. Nielsen and F. Barbaresco (Eds.): GSI 2013, LNCS 8085, pp. 7857793 2013.

(C) Springer-Verlag Berlin Heidelberg 2013 


$$
\begin{aligned}
& \mathfrak{h}\left(a_{1}, a_{2}\right)=\left(\frac{a_{1}^{-1}+a_{2}^{-1}}{2}\right)^{-1}, \\
& \mathfrak{a}\left(a_{1}, a_{2}\right)=\left(\frac{a_{1}+a_{2}}{2}\right), \\
& \mathfrak{g}\left(a_{1}, a_{2}\right)=\sqrt{a_{1} a_{2}} .
\end{aligned}
$$

Then one can prove 2,3 that Algorithm 1 converges quadratically to the geometric mean.

Algorithm 1. Let $a_{1}, a_{2}$ be two positive numbers

$$
\begin{aligned}
& \text { while } a_{1} \neq a_{2} \\
& \text { - } b_{1}=a\left(a_{1}, a_{2}\right) ; \\
& \text { - } b_{2}=\hbar\left(a_{1}, a_{2}\right) ; \\
& \text { - } a_{1}=b_{1} ; a_{2}=b_{2} ;
\end{aligned}
$$

end

\subsection{The Matrix Setting}

Let $A_{1}, A_{2}$ be positive definite (positive for short) matrices and let us define the harmonic, arithmetic, and geometric means as follows:

$$
\begin{aligned}
\mathcal{H}\left(A_{1}, A_{2}\right) & =\left(\frac{A_{1}^{-1}+A_{2}^{-1}}{2}\right)^{-1}, \\
\mathcal{A}\left(A_{1}, A_{2}\right) & =\left(\frac{A_{1}+A_{2}}{2}\right), \\
\mathcal{G}\left(A_{1}, A_{2}\right) & =A_{1}^{1 / 2}\left(A_{1}^{-1 / 2} A_{2} A_{1}^{-1 / 2}\right)^{1 / 2} A_{1}^{1 / 2}=A_{1}\left(A_{1}^{-1} A_{2}\right)^{1 / 2} .
\end{aligned}
$$

In 4 it was proved that the algorithm proposed in Section 2.1, replacing the positive scalars by matrices, converges to the matrix geometric mean.

\section{Beyond Two Variables}

In fact, there are quite some possibilities in generalizing the deduction of the geometric mean based on only using the arithmetic and harmonic mean to more than two variables. In this section, some straightforward, as well as more advanced iterative algorithms will be proposed.

\subsection{Circular Mean}

Consider a sequence of matrices, whose "geometric" mean we wish to compute. A simple, but effective and convergent procedure, as discussed in [5], is the following. 


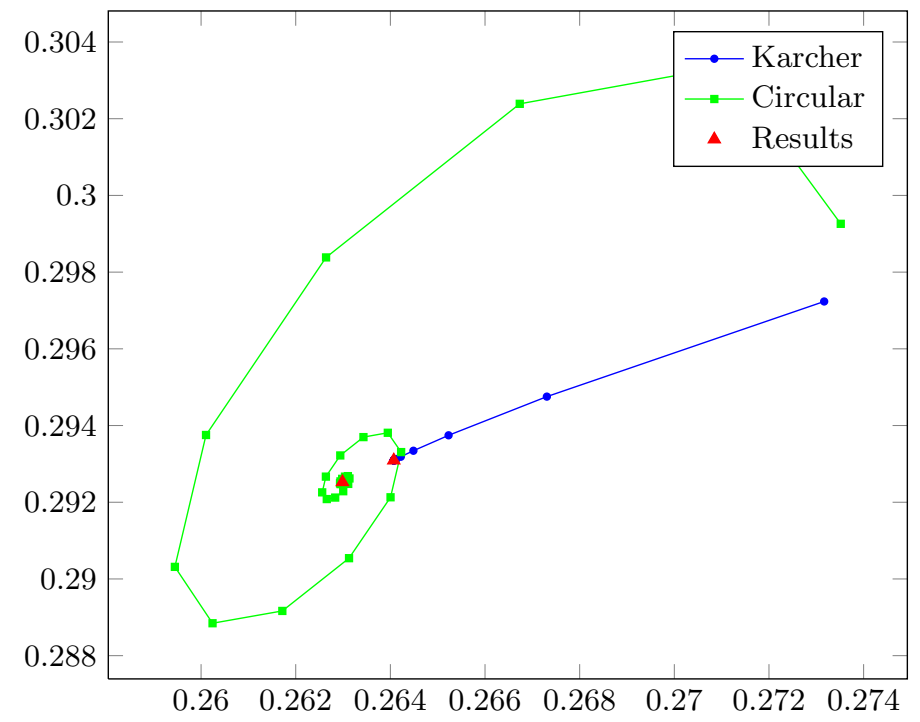

Fig. 1. Simplified representation of the iterations of the Circular mean (Section 3.1) and the Karcher mean. The results of both methods are clearly different.

Algorithm 2. Let $A_{1}, \ldots, A_{n}$ be $n$ positive matrices

while not converged

- For all $i$ set $B_{i}=\mathcal{G}\left(A_{i}, A_{(i \bmod n)+1}\right)$;

- For all $i$ set $A_{i}=B_{i}$.

end

This procedure was first proposed in [6], and a conjecture was made that this mean was possibly converging to the Karcher mean. However, this was afterwards proven to be false, as shown in Fig. 1.

Though this procedure neatly converges to an approximate mean, its convergence is rather slow. This is explained in 7, where it is proven that an iteration like this one on the corners of a random polygon converges to an elliptic setting. Obviously, once an ellips is reached, size reduction of the ellips, and convergence, becomes rather slow.

Let us graphically illustrate by an example that this behavior also occurs when considering Algorithm 2, The convergence towards this elliptic behavior is displayed in Fig. 2 using the consecutive eigenvalues in the iterations of the Circular mean of ten $2 \times 2$ matrices. Figure 2(b) clearly shows this slow behavior with iterations 200 and 201. The spiraling convergence of the Circular mean in Fig. 1 is another indication of this phenomenon. 


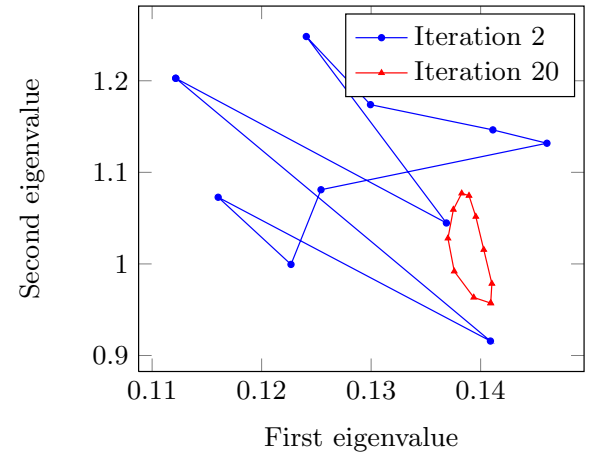

(a) Iteration 2 and 20 .

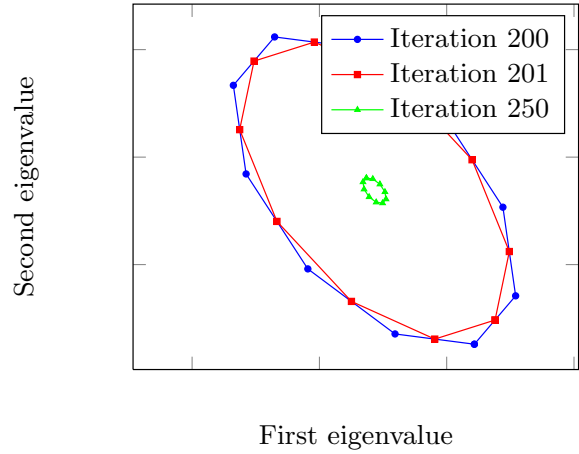

(b) Iteration 200, 201, and 250 .

Fig. 2. The evolution of the eigenvalues of ten $2 \times 2$ matrices over the iterations of the Circular mean (Section 3.1)

A simple, though effective remedy, is to randomly permute the matrices.

Algorithm 3. Let $A_{1}, \ldots, A_{n}$ be $n$ positive matrices

while not converged

- For all $i$ set $B_{i}=\mathcal{G}\left(A_{i}, A_{(i \bmod n)+1}\right)$;

- For all $i$ set $A_{p(i)}=B_{i}$, with $p$ a random permutation of $[1, \ldots, n]$.

end

Of course, this algorithm will never give the exact same result because of the random permutations. However, as shown in Fig. 3, the results can be found in the vicinity of the fixed-order result (the result obtained from Algorithm 2, without randomizing). Another interesting observation is that all the (randomized) results appear in a plane (Fig. [3 (b)], as mentioned for fixed order in 7 .

\subsection{Crude Midpoint Guess}

Instead of continuing the iteration process with $n$ matrices, one can reduce them right from the start to two.

Algorithm 4. Let $A_{1}, \ldots, A_{n}$ be $n$ positive matrices

- $B_{1}=\mathcal{A}\left(A_{1}, \ldots, A_{n}\right)$

- $B_{2}=\mathcal{H}\left(A_{1}, \ldots, A_{n}\right)$;

The solution is $\mathcal{G}\left(B_{1}, B_{2}\right)$.

The result of this somewhat simplistic algorithm will be a very rough approximation to the Karcher mean. However, it is very cheap to compute and can be seen to preserve some of the desired properties of geometric means, such as inversion invariance (the mean of the inverses is the inverse of the original mean). 


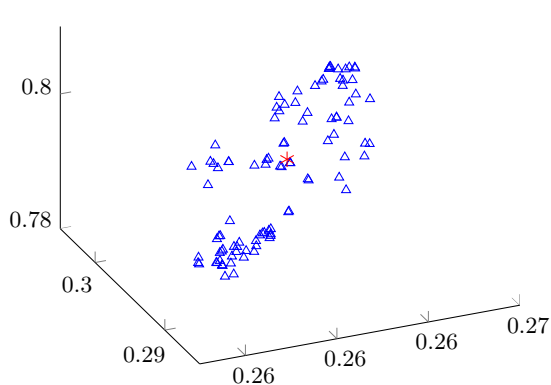

(a) The results of the random algorithm are close to the result of the fixed order algorithm.

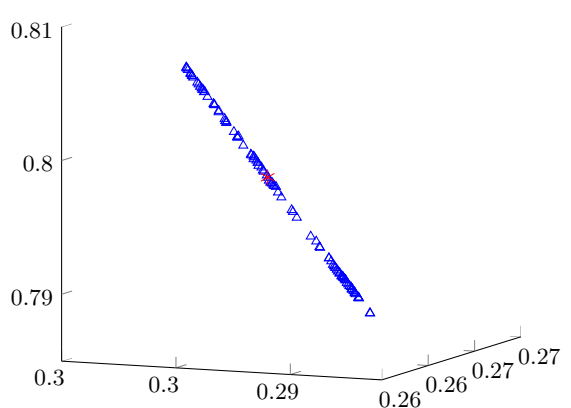

(b) The results (both randomized and fixed order) lie within the plane mentioned in [7].

Fig. 3. Results of the fixed order Circular mean (Algorithm 2) and 100 runs with the randomized Circular mean (Algorithm 3) for the same ten $2 \times 2$ matrices. The two figures display the same results, but for a different angle of view.)

\subsection{A Harmonic and Arithmetic Circular Iteration}

Since the original Algorithm 1 continuously combines the arithmetic and harmonic means, it would be interesting to generalize this towards more than two matrices. The next algorithm gives a possibility for such a generalization. We start by duplicating the set of matrices, one focusing on the harmonic and one on the arithmetic mean. These two sets are then iteratively combined in arithmetic and harmonic operations.

Algorithm 5. Let $A_{1}, \ldots, A_{n}$ be $n$ positive matrices

- For all $i$ set $B_{i}=A_{i}$ and $C_{i}=A_{i}$;

while not converged

- For all $i$ set $\tilde{B}_{i}=\mathcal{H}\left(B_{i}, C_{(i \bmod n)+1}\right)$;

- For all $i$ set $\tilde{C}_{i}=\mathcal{A}\left(B_{i}, C_{(i \bmod n)+1}\right)$;

- For all $i$ set $C_{i}=\tilde{C}_{i}, B_{i}=\tilde{B}_{i}$.

end

When experimenting with this algorithm, we notice that the vertices of the two sets $B_{i}$ and $C_{i}$ converge fast towards each other. Afterwards, the nearly equal sets tend to exhibit the elliptic behavior again, resulting in a slow total convergence. As before, we try to resolve this using a random permutation of the order of the matrices (see Algorithm 3). These results display the same behavior we noticed in Section 3.1, meaning that the results of the randomized algorithm all appear in the same plane and in the vicinity of the fixed order result.

\subsection{Symmetric Function Means}

In 8], two different means are presented based on an electrical networks approach, which are referred to as the T-mean and P-mean. For both means, when 
there are $n$ matrices, equally many positive functions $T_{i, n}$ or $P_{i, n}, i=1, \ldots, n$, are constructed and used to create a new $n$-tuple of matrices in each iteration. What makes these functions interesting in our context is that the functions $T_{1, n}$ and $P_{1, n}$ are the same and are equal to the arithmetic mean. The functions $T_{n, n}$ and $P_{n, n}$, on the other hand, are both equal to the harmonic mean. Furthermore, each of the functions is constructed using operations reminiscent of the arithmetic and harmonic mean (serial and parallel sums). Below we show the algorithm for the T-mean. The algorithm for the P-mean is completely similar and for the definition of the functions $T_{i, n}$ and $P_{i, n}$ we refer to [8].

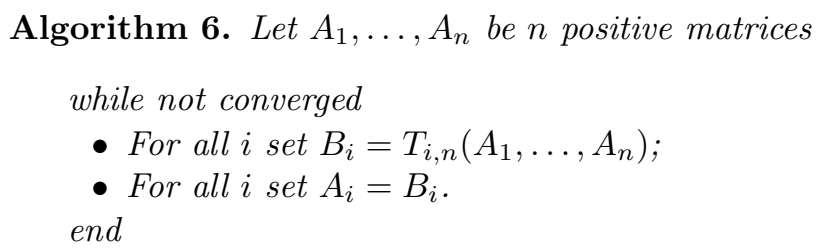

\section{Numerical Experiments}

In this section we will present numerical experiments to give more numerical insight in the behavior of all these algorithms. Both the speed of the algorithms and the proximity of their results to the Karcher mean will be discussed.

Afterwards, we test whether the proximity of the various algorithms to the Karcher mean makes them a good initial guess to start the manifold optimization. As a comparison, we also consider some standard initializers, namely the arithmetic, harmonic and cheap mean [9].

\subsection{Distance to the Karcher Mean and Computational Speed}

First, we compare the difference in performance between those algorithms which operate with a fixed order and their counterparts where the graph, which determines the sequence of the matrices, is randomized in each iteration. This concerns the fixed order version and randomized version of the algorithms in Sections 3.1 and 3.3 .

In terms of proximity to the Karcher mean, all four algorithms give nearly identical results. In Fig. 4(a), we display these results for the two versions of the algorithm in Section 3.3. For reasonable condition of the matrices, this proximity decreases only slightly as the condition of the matrices deteriorates. In terms of computational time, however, there is a clear difference. As can be seen in Fig. 4l(b) the algorithm in Section 3.3 is faster when using a randomized graph than with a fixed order. A very similar acceleration could be seen for the Circular mean (Section 3.1).

When comparing the algorithms of the two Sections, it is clear that the algorithm from Section 3.3 outperforms the Circular mean, since it has comparable proximity to the Karcher mean and requires less computational time. 


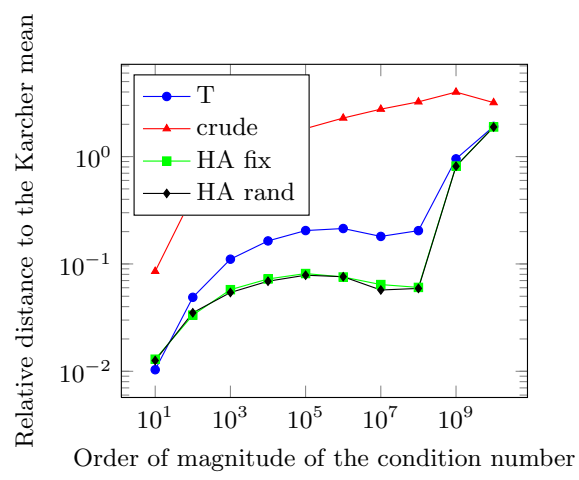

(a) Relative distance of some of the suggested means to the Karcher mean where the condition number of the matrices is varied. The figure displays only an average of such distances to avoid clutter. In the legend, $T$ indicates the T-mean, crude the mean in Section 3.2 , and $H A$ the mean of Section 3.3, both for fixed order and a randomized graph.

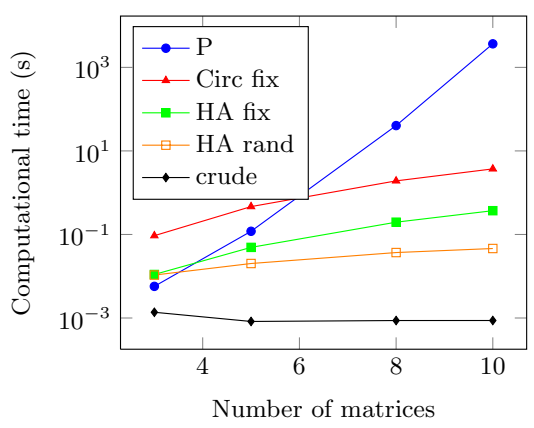

(b) Computational time of some of the suggested means for a varying number of matrices. The figure displays only an average of such timings to avoid clutter. In the legend, $P$ indicates the $\mathrm{P}$ mean, Circ fix the mean in Section 3.1 with fixed order, $H A$ the mean in Section 3.3, and crude the one in Section 3.2.

Fig. 4. Numerical properties of the various means

As for the T- and P-mean, we observe a proximity to the Karcher mean which is slightly worse than for the previous methods. However, the functions $T_{i, n}$ and $P_{i, n}$ are recursively defined, which causes the required computational time for these means to increase rapidly as the number of matrices starts to grow significantly large.

Finally, the crude guess from Section 3.2 behaves as its name suggests. It has a low computational time, but it also has a bad proximity to the Karcher mean.

In Fig. 5] we display the location of the various means with respect to the original matrices for a simple example of three positive $2 \times 2$ matrices. We added the geodesics, the lines of shortest distance in the Riemannian space, between these original matrices. It is interesting to show these since the non-Euclidean geometry of the space of positive matrices is the cause of the computational challenge of the Karcher mean.

\subsection{Initial Guesses for the Karcher Mean Algorithm}

After analyzing the various proposed means in terms of performance, we now test their appropriateness as an initial guess to the Conjugate Gradient (CG) algorithm for computing the Karcher mean. In Table1, the number of iterations the CG algorithm requires until convergence are shown for good conditioned and bad conditioned matrices. These results are rounded averages of a number of repetitions of the test.

In general, the required number of iterations is lower as the condition of the matrices becomes worse. However, this is an observation that is related to the dynamics of the Karcher mean, and not as much to the initial guesses, so we will not discuss this in these notes. 
Table 1. Number of iterations required for the Conjugate Gradient algorithm for the Karcher mean to converge, using the specified mean as an initial guess (averaged over some repetitions). $H A$ refers to the mean in Section 3.3

\begin{tabular}{|c||c|c|}
\hline condition number & $1 \mathrm{e} 1$ & $1 \mathrm{e} 8$ \\
\hline \hline Circular, fixed & 15 & 14 \\
\hline Circular, random & 15 & 15 \\
\hline Crude guess & 18 & 17 \\
\hline HA, fixed & 14 & 11 \\
\hline HA, random & 16 & 13 \\
\hline T-mean & 16 & 15 \\
\hline P-mean & 18 & 17 \\
\hline Arithmetic mean & 19 & 16 \\
\hline Harmonic mean & 21 & 17 \\
\hline CHEAP mean & 15 & 16 \\
\hline
\end{tabular}

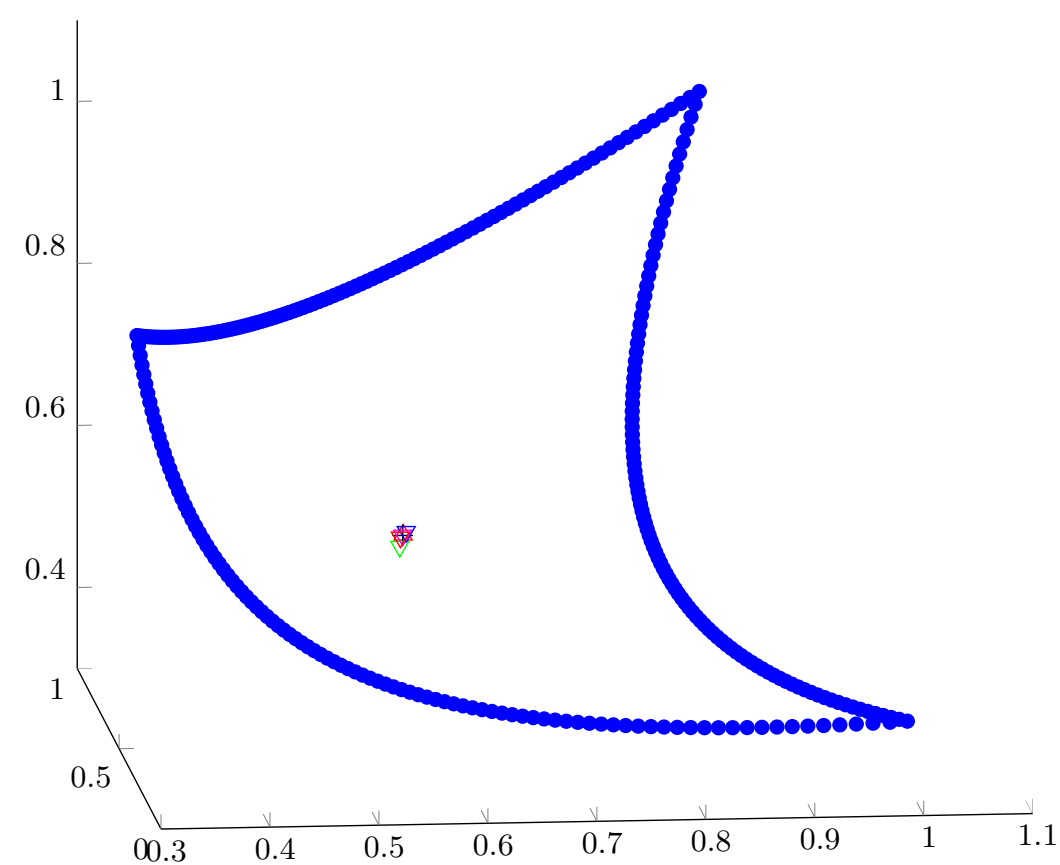

Fig. 5. Representation in 3D space of three positive $2 \times 2$ matrices. The curved triangle represents the geodesics, the lines of shortest distance, between the three matrices, which are the vertices. As can be seen, the various means appear very close to the Karcher mean in this simple example of well-conditioned matrices. 
After inspecting the table, we can see that in general both the CHEAP mean and the two fixed order means (Circular and HA) result in a low number of iterations. On the other hand, the arithmetic and harmonic mean, and the crude guess appear to give the worst results, and are therefore least suited as an initial guess to the Karcher mean. If we look at the bad conditioning separately, we also notice that the mean of Section 3.3 (HA) performs especially well, both with fixed order and randomized graph.

\section{Conclusion}

There is no fixed standard on how to generalize the arithmetic-harmonic algorithm for the geometric mean towards more than two matrices. We have examined some existing approaches and newly developed algorithms, judging their performance based on computational speed and proximity to the Karcher mean. A remarkable observation is that when working with algorithms that depend on a particular order of the matrices, randomizing this order in every iteration provides a significant speed-up while maintaining the proximity to the Karcher mean. Condering proximity to the Karcher mean, computational speed, and appropriateness as an initial guess to the Karcher mean optimization problem, the algorithm in Section 3.3 appears to provide optimal results.

Acknowledgements. The authors would like to thank Dario A. Bini for the interesting discussions during the Structured Matrix Days in Limoges, 2012; and Bruno Iannazzo and Pierre-Antoine Absil for their useful comments.

\section{References}

1. Ando, T., Li, C., Mathias, R.: Geometric means. Linear Algebra and its Applications 385, 305-334 (2004)

2. Borwein, J., Borwein, P.: Pi and the AGM: a study in the analytic number theory and computational complexity. Wiley-Interscience (1987)

3. Foster, D., Phillips, G.: The arithmetic-harmonic mean. Mathematics of Computation 42, 183-191 (1984)

4. Atteia, M., Raissouli, M.: Self dual operators on convex functionals; geometric mean and square root of convex functionals. Journal of Convex Analysis 8(1), 223-240 (2001)

5. Palfia, M.: A multivariable extension of two-variable matrix means. SIAM Journal on Matrix Analysis and Applications 32(2), 385-393 (2011)

6. Paige, C.: A useful form of unitary matrix obtained from any sequence of unit 2norm n-vectors. SIAM Journal on Matrix Analysis and Applications 31(2), 565-583 (2009)

7. Elmachtoub, A., Van Loan, C.: From random polygon to ellipse: an eigenanalysis. SIAM Review 52(1), 151-170 (2010)

8. Anderson, W., Morley, T., Trapp, G., et al.: Symmetric function means of positive operators. Linear Algebra and its Applications 60, 129-143 (1984)

9. Bini, D., Iannazzo, B.: A note on computing matrix geometric means. Advances in Computational Mathematics 35, 175-192 (2011) 